\title{
Chromatin texture from hematoxylin stained thyroid lesions
}

\author{
Olga Ferrer-Roca, José A. Pérez Gómez and Maritza Estévez \\ Grupo Texcan, Cátedra Anatomía Patológica, Facultad de Medicina, Universidad de la Laguna, \\ 38071 Tenerife, Canary Islands, Spain \\ Tel.: +34 22 642015; Fax: +34 22 641855; E-mail: catai@ redkbs.es
}

Received 2 June 1997

Revised 18 November 1998

Accepted 26 January 1999

\begin{abstract}
Quantitative aspects of cytology and histology should be considered in diagnostic standardisation processes. The present paper summarises the cytological differences detected in 75 thyroid lesions using a computerized textural analysis.

Cells stained with progressive hematoxylin and taken from paraffin blocks were overlaid with the extracted texture. This technique was based on the lineal detection of a grey level gradient of the common logarithm of the integrated optical density (IOD) of each nucleus.

Diffuse and nodular goiters (36 cases) were demonstrated to be composed of small cells containing high density texture that, on microscopical visual inspection, gave a "salt and pepper" appearance. The adenomatous goiters ( 2 cases) and adenomas (26 cases) were composed of low texture cells with a visual "blurry or smudgy" chromatin, while the atypical adenomas with capsular invasion (4 cases) were characterised by a "woodworm" nuclear appearance that produced the highest texture of the series. Finally, encapsulated folliculo-papillary carcinomas (3 cases) were composed of large clear nuclei with high IOD, low texture, and scattered lines that resulted in an "empty grape skin" aspect.

Our findings seam to confirm the suitability of computerized textural techniques that aid in recognizing cell microscopic features objectively. The one used in the present work, based on a mathematical function of the DNA content of each individual nucleus (IOD), fulfills all microscopy detection criteria.
\end{abstract}

Keywords: Image analysis, texture, chromatin, thyroid, cytology, benign tumours

\section{Introduction}

Chromatin diagnostic features in pathology may be recognised by computer. Nevertheles, computer assisted textural analysis may also detect differences which are difficult to assess by microscopy. The experiment described in this paper was designed to detect chromatin texture characteristics of cell components in a group of thyroid lesions, most of them benign.

Some of the problems linked to cell texture analysis are summarized and grouped by the following points: (1) Simultaneous cell texture analysis and DNA quantification in Feulgen stained nuclei poses difficulties. The pre-treatment with RNAasa and the hydrochloric acid $(\mathrm{HCl})$ denaturation affects the chromatin pattern on which pathologists base their diagnoses (Giemsa and Hematoxylin in thyroid). Conversely, on thyroid tissue, chromatin distortion induced by paraffin-embedding has an added diagnostic value for detecting papillary cells or colloid reabsorption vacuoles. (2) Certain problems arise from the chosen parameters and techniques; some mathematical models do not have a visual perception counterpart (reviews $[8,14]$ and [18]). For example, demonstrating that the Skewness of the optical 
density histogram differenciates adenomas from carcinomas of the thyroid $[15,16]$ is of limited value to cytologists. (3) There are some difficulties in reproducing results, except in well standardised series [4]. A texture analysis applied to cells modified by fixation and processing (point 1), requires parameters as independent as possible from the cell morphometry $[2,17]$. Use of image analysis stardardisation techniques with median filters followed by linear scaling of grey levels [18], may also read to complications.

These arguments guided the design, in 1989, of a computer programme $[9,11]$ for detecting lineal ${ }^{1}$ grey level differences based on the common logarithm of the integrated optical density (IOD) of each nucleus which has been shown to stress the differences in thyroid cells. The thyroid tissue is composed of a few c-type cells and numerous follicular ones; in pathological conditions the latter are transformed into "papillary" cells characterized by a distinctive chromatin texture with grooved clear nuclei of high IOD [10]. Our computer programme was designed to produce a visual textural image on the grey level transition points to help pathologists interpret chromatin texture and detect morphological findings that may be of diagnostic and prognostic significance; this is a relevant problem in borderline lesions, or in atypical adenomas of the thyroid.

Applying the previous technique to a group of 75 thyroid lesions, most of them hyperplastic and benign, we could recognised some distinctive cell components in various histological subtypes that might be of help in cytological screening.

\section{Material and methods}

This research was based on the textural analysis of 100 cells per case in 75 thyroid lesions. Among them 37 goiters: 5 diffuse hyperplastic (DHG), 30 nodular colloid (NCG), 2 nodular adenomatous (NAG); 27 adenomas: 18 simple adenomas (SA); 2 atypical adenomas (AA); 4 atypical adenomas with capsular invasion (AAI); 3 Hürthler adenomas (AH), together with 4 normal thyroids (nT), and 3 folliculo-papillary carcinomas (FPC). Four cases contained mixed lesions: 1 NCG together with a sclerosing follicular carcinoma; 1 case of SA besides thyroiditis, 1 SA plus a Hürthler adenoma, and 1 AA simultaneous with a follicular carcinoma.

The diagnosis and case classification were based on the histology of the samples applying the following criteria: DHG with or without lymphoid infiltrates; the former were Graves' diseases cases. NAG were nodular goitres with solid and cellular nodular lesions similar to foetal or embrionary adenomas, but without capsule. Adenomas included only single encapsulated lesions; among them the AA were those with atypical cells and mitosis; the AAI presented an additional invasion of the capsule. The FPC were invasive follicular encapsulated lesions with clear nuclei or papillae in some areas (it has to be mentioned that two of them had been initially miss-classified as follicular carcinomas).

Cytological specimens were obtained in all cases from paraffin-blocks following the technique of Hedley [7]. Cytospin preparations were stained with progressive hematoxylin [6,9], a semi-stoichiometric staining that allows DNA evaluation [13]. The term "semi-stoichiometric staining" is applied to the modified formula of progressive hematoxylin of Carazzi [13] whose final stain is proportional to the DNA content, although not dependent upon a selective fixation on purine-deoxyribose bond, thus producing histograms with slightly higher coefficient of variation in the modal DNA peak when compared with Feulgen stain.

\footnotetext{
1"lineal" means consecutively, i.e., one pixel after another, generally following the X-axis (the term has neither a statistical linear meaning nor refers to a linear disposition).
} 
Individual cells were selected (100 cells per case) with an Olympus BH2 microscope with a $60 \times$ oil Plan Apochromatic objective, an intermediate lens of $2.5 \times$, as well as an Aplanatic Achromatic condenser, both of 1.4 numerical aperture. A Sony CCD one chip B/W camera was used to digitize the cells into the Texcan system [11]. The system is based on a Matrox PIP-1024 digitizing board and cell images were processed by our own software running PC compatible computers under MS-DOS.

Texture feature extraction, as well as the linear discriminant analysis automatically classifying the cells, are described in detail elsewhere [8], being summarised herein. The technique encloses two linear discriminants: the discrimant 1 is based on the parameter of Integrated Optical Density (IOD) and on the number of pixels detected with the grey level gradient, being highly sensitive to papillary cells; the discriminant 2 is a function of the number of pixels belonging or not to the grey level gradient region and is particularly sensitive to follicular cells. Pixels pertaining to a texture gradient region were those in which the grey level gradient, as a function of the common logarithm $(\log 10)$ of the individual cell IOD, was detected with an $8 \times 8$ mask applied in one direction.

Cells analysed with the above technique [8-11] were individually classified into follicular or papillary type. While processing, the analysed of studied cells were stored in computer files as the ones shown in the Figs 1 and 2.2

The analysis of each computer extracted parameter was used as a guideline for visual perception and cell feature identification. For each cell, those parameters known to demonstrate a visual perception under microscopy were recorded. They included: number of pixels belonging or not to the texture gradient either as direct value or normalised by the area of the nucleus; mean optical density (MOD); the standard deviation of the OD (sdOD); the integrated optical density (IOD) and the area of the nucleus together with the number of fragments with texture (consecutive pixels with textural gradient).

Correlation of the majority of previous parameters has already been shown elsewhere [8] being excluded in the multivariant linear discriminant analysis technique. Only those included in the discriminant 1 and 2 were not correlated.

Case summary statistics of the individual cell parameters were mean values and true standard deviation. Statistics were applied to every histological subgroup for comparison purposes, or to each case for correlation purposes. Parameters were grouped as follows: Cell composition refers to the percentage of papillary cells in discriminant 1 . Morphometry alludes to the mean nuclear area (A) and size variation per case as evaluated with the sd of the area (sdA). Densitometry refers to the mean OD (MOD) as well as to the IOD per nucleus. Among the textural parameters we included the sdOD per nucleus (since it expresses the degree of grey level transition in chromatin), the automatic texture analysis with the number of pixels having a grey level gradient (Tpx), as well as those pixels without gradient with their sourrounding ones (not-Tpx), the latter normalised or not by the area of the nucleus (Tpx/A); and finally, the number of fragments having consecutive pixels with grey level gradient detection (LTF).

The two tail $t$-Student test for comparison of the mean was applied to quantitative parameters. The results were used to validate the microscopical differences of chromatine distribution in the various histological subgroups. Bonferoni's $t$-test was used to detect the differences when several variables were considered simultaneously. Correlation between the mean of quantitative parameters and the number of papillary cells (in percentage) per case, was carried out with the linear Pearson test.

\footnotetext{
${ }^{2}$ Note that the original images are in black because the programme uses optical density images (obtained from a pixel by pixel computation between the original image and a similar one from the background of the whole setting) to evaluate their texture; these optical density images were digitally inverted to facilitate its comprehension to cytologists, while texture images were left as originally.
} 
Table 1

Summary of the quantitative parameters among the histological subgroups expressed as mean (sd)

\begin{tabular}{|c|c|c|c|c|c|c|c|c|c|}
\hline $\begin{array}{l}\text { Histology } \\
(n \text { cases) }\end{array}$ & $\begin{array}{c}\text { DHG } \\
(5)\end{array}$ & $\begin{array}{c}\text { NCG } \\
(30)\end{array}$ & $\begin{array}{c}\text { NAG } \\
\text { (2) }\end{array}$ & $\begin{array}{l}\text { SA } \\
(20)\end{array}$ & $\begin{array}{l}\text { AA } \\
\text { (3) }\end{array}$ & $\begin{array}{l}\text { AAI } \\
(4)\end{array}$ & $\begin{array}{c}\mathrm{AH} \\
(3)\end{array}$ & $\begin{array}{l}\mathrm{nT} \\
\text { (4) }\end{array}$ & $\begin{array}{c}\text { FPC } \\
(3)\end{array}$ \\
\hline 6 nan & 27 (33) & $26 \quad 3$ & $75 \quad(1$ & $\begin{array}{ll}59 \quad(27\end{array}$ & $47 \quad 3$ & $4 \quad(2$ & $75 \quad(18$ & $43 \quad 3$ & $61 \quad 2$ \\
\hline & $6(151)$ & 905 (145) & 106 (134) & $976(163)$ & 929 (161) & 917 (175) & 20) & 1005 (329) & 1151 \\
\hline $\operatorname{sdA}$ & $9 \quad(62)$ & 157 (39) & $45 \quad(10)$ & 171 & 192 (24) & 182 (39) & $240 \quad(25)$ & 153 & 155 (19) \\
\hline DI & $78(155)$ & 838 (239) & $1282(282)$ & $920(232)$ & 762 (157) & $823(268)$ & 1217 (139) & 643 (179) & 986 (118) \\
\hline & $7 \quad(83)$ & $136 \quad(51)$ & $133 \quad(39)$ & 144 & $174(65)$ & 144 (28) & $251 \quad(5$ & 93 & 111 \\
\hline MOD & (16) & $94 \quad(22$ & $84 \quad(20)$ & 96 & 83 & $90 \quad(21)$ & 109 & $67 \quad(1$ & 88 \\
\hline & & 26 & 18 & 21 & 20 & 30 & 22 & & 13 \\
\hline & 49 (18) & 49 (17) & (2) & 34 & 38 & (2) & 33 & $42 \quad(2$ & 34 \\
\hline sdTpx & (6) & $105 \quad(32)$ & $94 \quad(22)$ & 105 & 92 (10) & 115 (22) & 125 (108) & $78 \quad(26)$ & (14) \\
\hline & 51 (18) & $51 \quad(17$ & (5) & $66 \quad(10$ & 63 & (2) & 68 & $58 \quad(21)$ & 66 \\
\hline & 15 (11) & 15 (12) & (3) & 23 (14) & (7) & $6(0.5)$ & (9) & 21 (14) & 21 \\
\hline LTF & 119 (13) & $121 \quad(30)$ & $131 \quad(22)$ & 117 (26) & 126 & 140 & $120 \quad(19)$ & 132 (42) & $162 \quad(30)$ \\
\hline
\end{tabular}

\section{Results}

Table 1 shows the quantitative parameters among the histological subgroups. As detailed in the previous section, the parameters were grouped into: percentage of cell types together with the mean of morphometry, densitometry, and textural parameters. The data were translated into visual perception (Figs 1 and 2) using graphic words in quotation marks.

The analysis of goiters $(G)$ - excluding NAGs - when compared with adenomas (A) demonstrated:

A cellular component with predominant "follicular" cell type $(p<0.005)$.

A morphometry with small $(p<0.05)$ and regular cells (sdAREA $p<0.025)$.

A densitometry with no differences.

A texture analysis with a high sdOD and Tpx/A $(p<0.05)$ forming the so called "salt and pepper" appearance (Fig. 1a).

Considering several simultaneous variables, only the type of cells and their regularity showed statistical differences (Bonferoni's $t$-test $p<0.03$ ) between $\mathrm{G}$ and A.

The analysis of NAGs group revealed:

A cellular component with predominant "papillary" cells $(p<0.0005$ versus $\mathrm{G})$ due to their absence of internal texture.

A morphometry with larger cells ( $p<0.05$ vs. G) and regular in shape (sdA).

A densitometry with the highest IOD of the series, being closer to AH and far from goiters $(p<$ $0.005)$ and adenomas $(p<0.05)$.

A texture analysis with a low sdOD and Tpx/A, distinctive from adenomas $(p<0.05)$ and goiters $(p<0.0005)$ and indicative of the "smudgy or blurry" appearance of the nuclei (Fig. 1b).

Considering several simultaneous variables the NAGs were different from goiters with regard to their cell component, nuclei IOD, sdOD, and Tpx/A and were statistically significant using Bonferoni's $t$-test $(p<0.007)$. 


\begin{tabular}{|c|c|c|c|c|}
\hline$\theta$ & 0 & 0 & (3) & 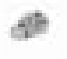 \\
\hline • & $a$ & te & อ & e \\
\hline$\bullet$ & 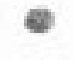 & 6 & b & 8 \\
\hline et & $\theta$ & $\theta$ & 9 & 6 \\
\hline 9 & $b$ & $e$ & 8 & 9 \\
\hline
\end{tabular}

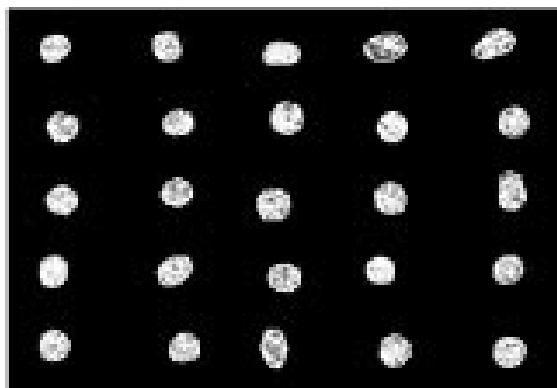

(a)
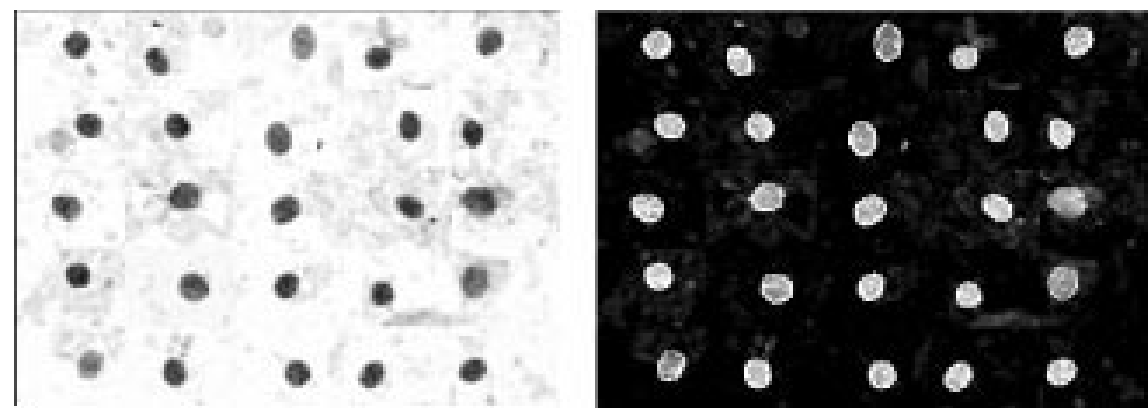

(b)
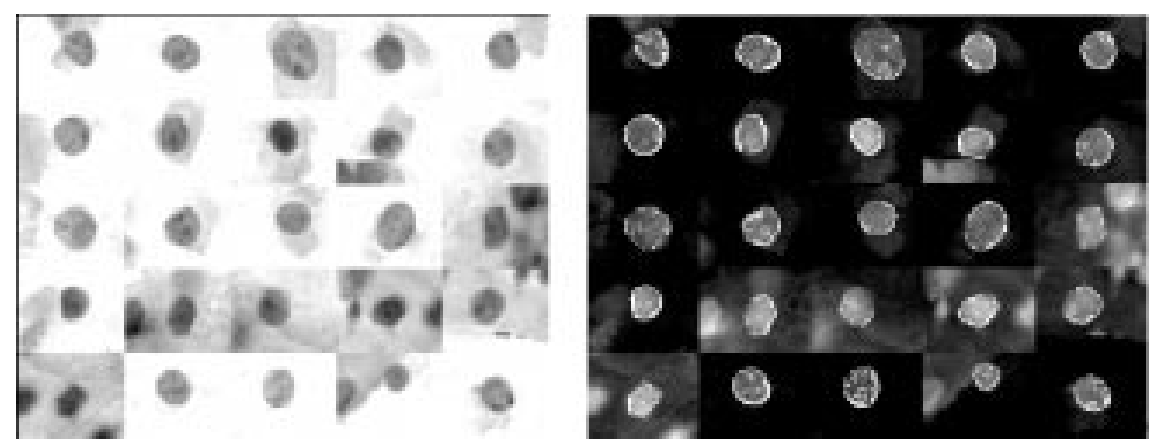

(c)

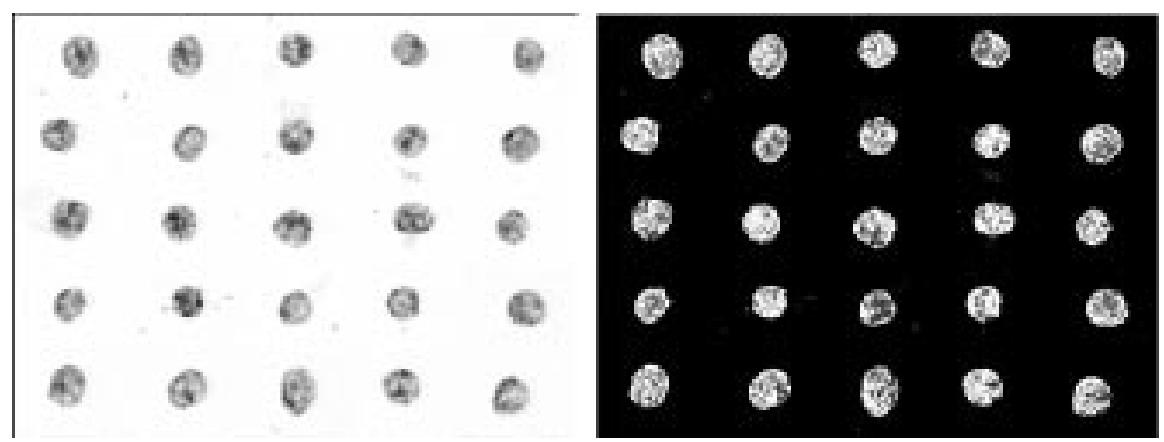

(d)

Fig. 1. Inverted optical density (OD) images on the left, compared with computer-detected lineal texture based on the log of the IOD, seen on the right. (a) - Goiter with follicular cells with "salt and pepper" chromatin. (b) - NAG of cells with high IOD giving in the texture (right) a papillary type cell showing the "blurry" chromatin aspect. (c) - HA integrated by cells with the "smudgy or blurry" aspect. (d) - AAI with the typical "wood-wormed" aspect that produces high texture. 

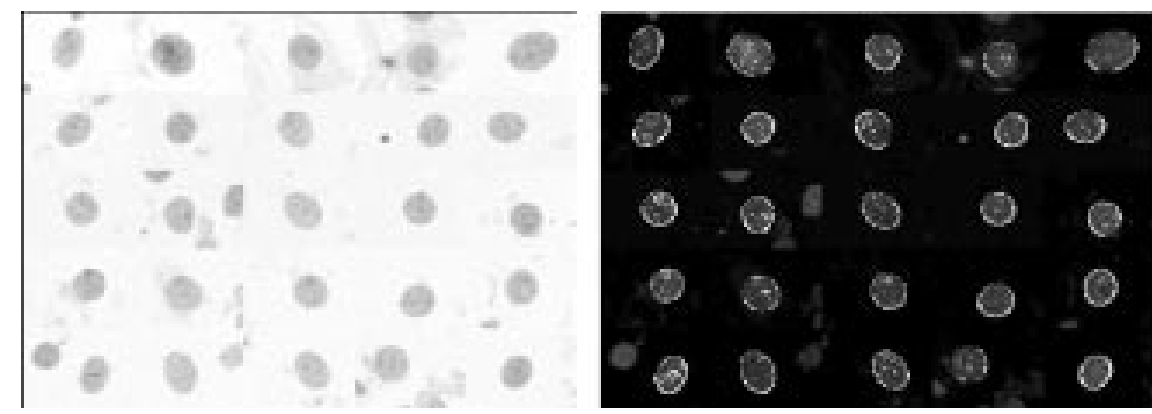

(a)
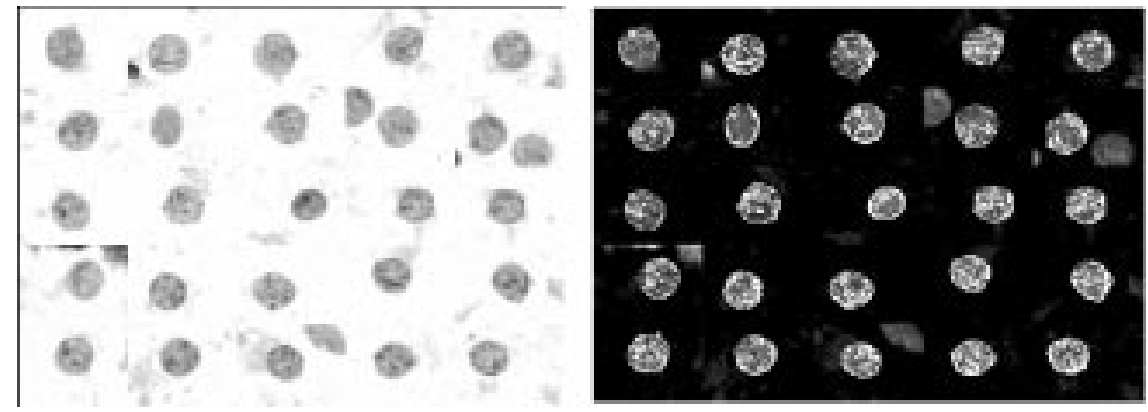

(b)

Fig. 2. Shows the two pathologies representing the greatest differences in the series (see legend Fig. 1). (a) - Normal thyroid with delicate chromatin and clear nuclei. (b) - FPC with big round cells of clear chromatin aspect due to nuclear membrane condensation with LTF. The LTF are specially apparent in the cell number 2 giving the aspect of "empty grape skin".

The study of the different subtypes of adenomas - excluding the AAI - demonstrated:

A cellular component with high number of "papillary" classified cells.

A morphometry with no differences, although AH had larger cells.

A densitometry with no differences, although AH had one of the highest IOD in the series.

A texture with low sdOD and low Tpx/A indicative of a "smudgy or blurry" appearance (Fig. 1c) of the nuclei.

By contrast, the atypical adenomas with capsular invasion (AAI) had to be separated from the previous group due to their distintive texture that showed:

A cellular component almost exclusively integrated by "follicular" cells ( $p<0.0005$ vs. G, A, FPC).

A morphometry with no statistical differences (ns).

A densitometry with no statistical differences.

A texture analysis with higher sdOD (ns) and a high Tpx/A $(p<0.0005$ vs. G, A, FPC) due to the "woodworm" aspect of their nuclei lying among the "salt and pepper" chromatin distribution (Fig. 1d).

Considering several simultaneous variables, the AAIs were clearly different from the rest of adenomas in relation to their cell component and high Tpx/A (Bonferoni's $t$-test $p<0.001$ ).

The greatest differences amonst the samples of our series were represented by the normal thyroid on the one hand and, the folliculo-papillary carcinomas on the other hand.

With regard to normal thyroids, 
The cellular component was in between goiters $(\mathrm{G})$ and adenomas (A).

The morphometry showed cells larger than goiters $(p<0.005)$, but of similar uniformity.

The densitometry revealed a low and uniform IOD ( $p<0.05$ vs. G and A) with a low MOD indicating the presence of "clear cells" $(p<0.0005 \mathrm{G}, p<0.005 \mathrm{~A})$.

The texture analysis had a low sdOD $(p<0.0005 \mathrm{G}$ and $p<0.05 \mathrm{~A})$ with low sdTpx indicative of a "fine" chromatin (Fig. 2a).

Cell size, low MOD, and low sdOD differentiated the normal thyroid from the goiters according to Bonferoni's $t$-test $(p<0.006)$.

In regard to the folliculo-papillary carcinomas,

The cellular component was of the "papillary" type ( $p<0.025$ vs. G).

The morphometry showed large uniform cells ( $p<0.005$ vs. G).

The densitometry revealed a high IOD and a medium value of MOD $(p<0.005$ and $p<0.01$, respectively, vs. normal thyroid).

The texture analysis $\quad$ showed a low sdOD ( $p<0.0005$ vs. G, $p<0.05$ vs. A) indicative of a uniform and low chromatin texture save for the presence of LTF that produced an "empty grape skin" appearance in some nuclei (Fig. 2b). The relationship between non-Tpx/Tpx was also clearly distinguishable from AAI $(p<0.0005)$.

Differences between FPC and AAI, considering several simultaneous variables, were based on cell component, Tpx/A, and non-Tpx/Tpx (Bonferoni $t$-test $p<0.002$ ).

In the analysis of the individual cases of the series, the correlation between cell component ( $\%$ cells) and mean values of the parameters demonstrated a high correlation with textural parameters Tpx/A and non-Tpx/Tpx, both with an $r=0.88$; a moderate correlation with sdDO $r=-0.52$ and area $r=0.5$, and low correlation with IOD $r=0.3$ or LTF $r=-0.3$. All correlations were statistically significant $(p<0.001$ or less).

\section{Discussion}

The computerised texture programme applied to the 75 thyroid lesions of the present series, mainly hyperplastic and benign, could be of value to identify statistically significant hallmarks in various histopathological subtypes.

The present technique seemed to overcome the three main problems outlined in the introduction: (1) It was applied to hematoxylin stained nuclei; (2) Location of the grey level gradient by computerised techniques had a direct visual correlation; (3) The parameters used in quantitative texture became morphometry independent when they were normalised by the nuclear area. Furthermore, the strategy to detect a gradient based on the common logarithm of the IOD of each nucleus, was in accordance with the changes in chromatin distribution depending on nuclear DNA and karyotype variations [16].

With this technique some objective chromatin texture variations, likely to be recognized through the microscope, were found: Normal thyroid with regular cell size and IOD showed clear nuclei (low MOD) and "fine" texture (low sdOD and sdTpx). Goiters were composed of small cells with "salt and pepper" chromatin (high sdOD and Tpx/A). Nevertheless, goiters containing adenomatous nodules (NAGs) were similar to adenomas due to their "blurry" cells with low texture (sdOD; Tpx/A) and high IOD; those cells identified as papillary by the computer programme were clearly different from FPC cells (Figs $1 \mathrm{~b}$ and 2b) sharing only a low texture and high IOD (see below). 
Adenoma cells (AAI excluded) were larger than goiter cells having low sdOD and texture, indicating that their nuclei do not have distinctive chromocenters, with "smudgy or blurry" heterochromatin. Hürthle adenomas had one of the highest densitometric values (IOD, MOD) of the series. Conversely, Atypical adenomas with capsular invasion (AAI) were composed by a different cell type that was closer to that of follicular cells of goiters (see Figs 3a and 3d); they showed a uniform size and distinctive chromatin characterized by a conspicuous heterochromatin with high transition grey levels towards the euchromatin areas; this texture produced a nuclear "woodworm" apearance very different from the FPC cell morphology.

Finally, FPCs were characterised by larger cells with high and uniform IOD in otherwise clear nuclei (low MOD and high not-Tpx/Tpx) containing the classical groves ("empty grape skin") detected with the parameter LTF (Fig. 2b). A special comment should be made to the expression "clear nuclei" applied to carcinomatous papillary cells, since the MOD (low compared with goiters or adenomas) is higher than that of normal thyroid $(p<0.05)$.

Whether the "smudgy or blurry" appearance of the chromatin in adenomas and NAGs is an intermediate stage between the "salt and pepper" follicular cell (goiter and AAI) and papillary "clear" cell (FPCs) is uncertain.

Finally, the analysis of the modal peak of DNA values in the various histological subtypes has already been analysed in a previous paper [12] together with the genetic polymorphism, polyploidization, trisomies, double clones, and other genetic markers associated to thyroid pathology.

In the present analysis we would like to stress the relevance of the quantitative aspects in the standardisation of diagnostic criteria both in benign and malignant thyroid lesions. For example, some cases of encapsulated follicular carcinomas with clear chromatin published in the literature [5] whose morphology resembles that of FPCs [1,3] by the size of their nuclei, low MOD and chromatin groves which are different from the atypical adenomas with capsular invasion (AAI) of the present series, whose cells had a chromatin texture closer to the follicular cells of goiters.

To conclude, we believe that the technique presented here has proved helpful in detecting some chromatin texture aspects of borderline (encapsulated FPC versus atypical follicular adenomas with capsular invasion) and benign-hyperplastic thyroid lesions (goiters and NAGs or adenomas and AAI). These changes in particular are recognized not only by a computer assisted programme, but they also could be observed by a cytology screener.

\section{Acknowledgements}

We would like to thank our technicians E. Gomez Ruiz and J. Spencer for their kind collaboration. Research supported by a grant No 9/91 from Canary Islands Government.

\section{References}

[1] J. Albores-Saavedra, E. Gould, C. Vardaman and F. Vuitch, The macrofollicular variant of papillary thyroid carcinoma. A study of 17 cases, Hum. Pathol. 22 (1991), 1195-1205.

[2] M.E. Boon and L.P. Kok, An explanation for the reported variability of nuclear areas in air-dried Romanowsky-Giemsastain smears of follicular tumors of the thyroid, Acta Cytologica 31 (1987), 527-530.

[3] K.T.K. Chen and J. Rosai, Follicular variant of thyroid papillary carcinoma. A clinicopathologic study of six cases, Am. J. Surg. Pathol. 1 (1977), 123-130.

[4] F. Collin, I. Salmon, I. Rahier, J.L. Pasteels, R. Heimann and R. Kiss, Quantitative nuclear cell image analyses of thyroid tumors from archival material, Hum. Pathol. 22 (1991), 191-196. 
[5] H. Galera-Davison, M. Bibbo, P.H. Bartels, H.E. Dytch, R. Gonzalez-Campora, F. Sanchez and G.L. Wied, Differential diagnosis between follicular adenoma and follicular carcinoma of the thyroid by marker features, Anal. Quant. Cytol. Histol. 8 (1986), 195-200.

[6] G.W. Gill, J.K. Frost and K.A. Miller, A new formula for a half oxidised hemathoxylin solution that neither overstains or requires differentiation, Acta Cytol. 18 (1974), 300-311.

[7] D.W. Hedley, M.L. Friedlander, I.W. Taylor, C.A. Rugg and E.A. Musgrove, Method for analysis of cellular DNA content of paraffin-embedded pathological material using flow cytometry, J. Histoch. Cytochem. 31 (1983), 1333-1335.

[8] O. Ferrer-Roca, E. Ballester-Guardia and J. Martin, Nuclear chromatin texture to differentiate follicular and papillary carcinoma of the thyroid, Path. Res. Pract. 185 (1989), 561-566.

[9] O. Ferrer-Roca, Software de analisis textural con aplicaciones citologicas, in: Analisis de Imagen (II), O. Ferrer-Roca, ed., Univ. La Laguna editions, Tenerife, 1990, pp. 81-117.

[10] O. Ferrer-Roca, E. Ballester-Guardia and J. Martin Rodriguez, Morphometric, densitometric and flow cytometric criteria for the automated classifcation of thyroid lesions, Anal. Quant. Cytol. Histol. 12 (1990), 48-55.

[11] O. Ferrer-Roca and J.A. Martin, Informatics in pathology. Software developed by the Texcan-group, in: Advances in Analytical Cellular Pathology, G. Burger, M. Oberholzer and G.P. Vooijs, eds, Excerpta Medica, International Congress series 911, Amsterdam, 1990, pp. 41-42.

[12] O. Ferrer-Roca, J.A. Perez Gomez, J. Cigurosa and E. Gomez, Genetic heterogeneity of benign thyroid lesions. Static and flow cytometry, karyotyping and "in situ" hybridisation analysis, Anal. Cell. Path. 16 (1998), 101-110.

[13] G.W. Gill, J.K. Frost and K.D. Miller, A new formulae for half oxidised hematoxylin solution that neither overstains or requires differentiation, Acta Cytol. 18 (1974), 300-311.

[14] P. Karakitsos, Cochand-Priollet and P. Guillausseau, Potential of the back propagation neural network in the morphologic examination of thyroid lesions, Anal. Quant. Cytol. Histol. 18 (1996), 494-500.

[15] F. Liautaud-Roger, J. Dufer, M. Pluot, M.J. Delisle and P. Coninx, Contribution of quantitative cytology in the cytological diagnosis of thyroid neoplasm, Anticancer Res. 9 (1989), 231-234.

[16] F. Liautaud-Roger, J.R. Teyssier, D. Ferrer, J. Dufer and P. Conninx, Can chromatin texture predict structural karyotypic changes in diploid cells from thyroid cold nodules?, Anal. Cell. Pathol. 4 (1992), 421-428.

[17] R.G. Wright and H. Castles, Variability of thyroid cell nuclear size with Romanowsky stains, Acta Cytologica 31 (1987), 526-527.

[18] K. Yogesan, T. Jorgensen, F. Albregtsen, K.J. Tveter and H.E. Danielsen, Entropy-based texture analysis of chromatin structure in advanced prostate cancer, Cytometry 24 (1996), 267-268. 


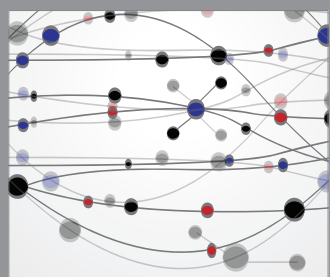

The Scientific World Journal
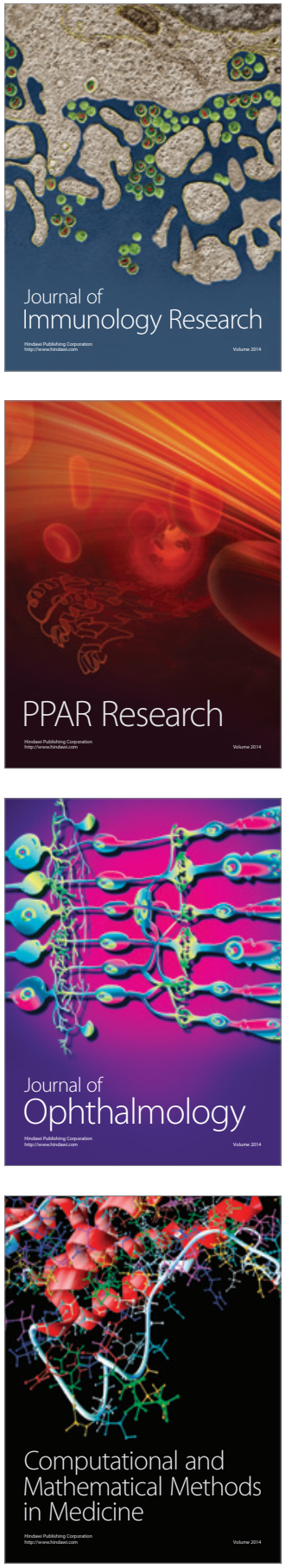

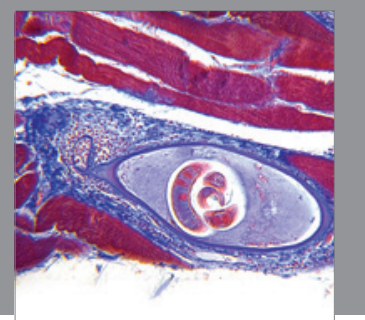

Gastroenterology

Research and Practice
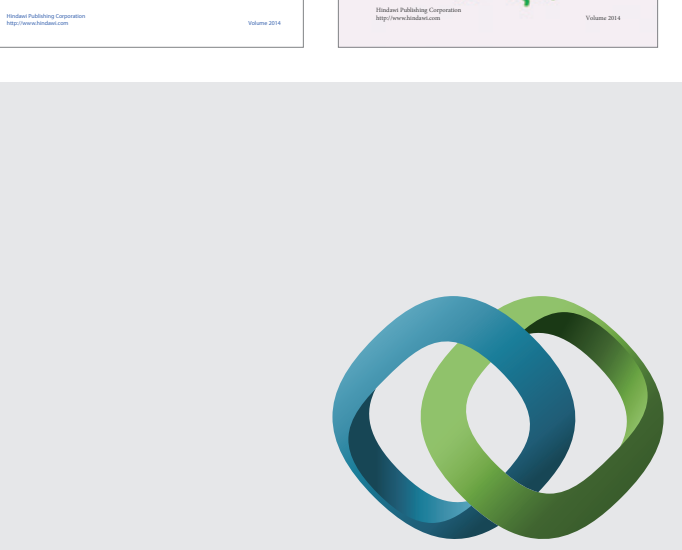

\section{Hindawi}

Submit your manuscripts at

http://www.hindawi.com
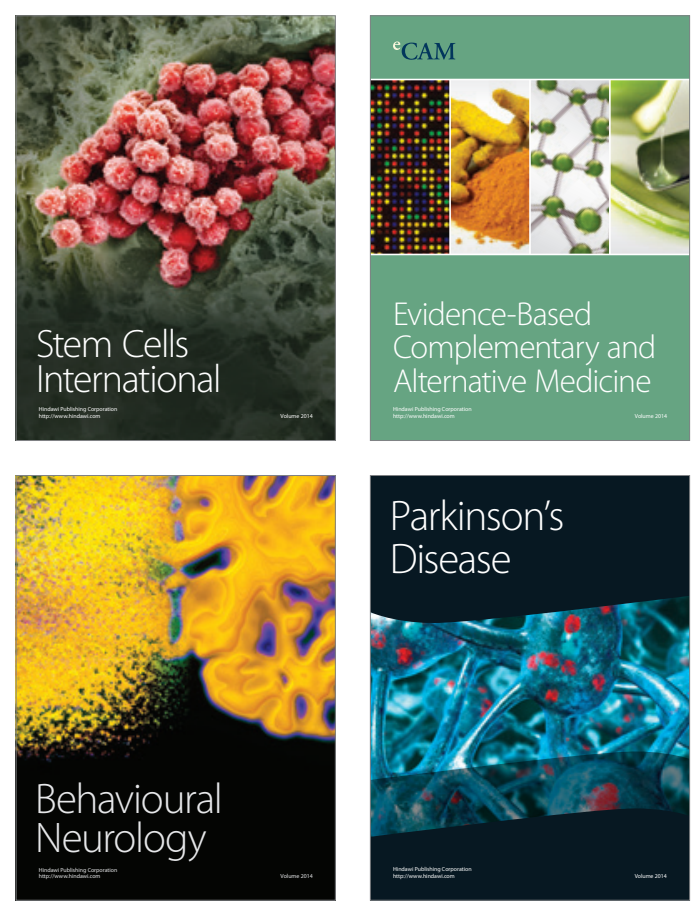

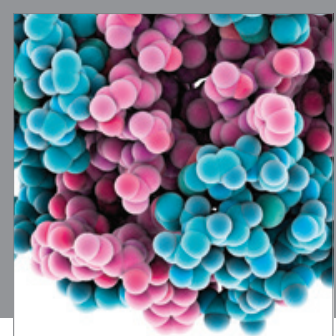

Journal of
Diabetes Research

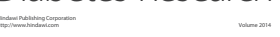

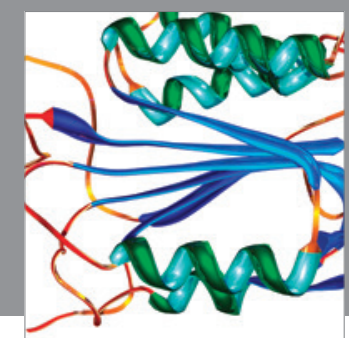

Disease Markers
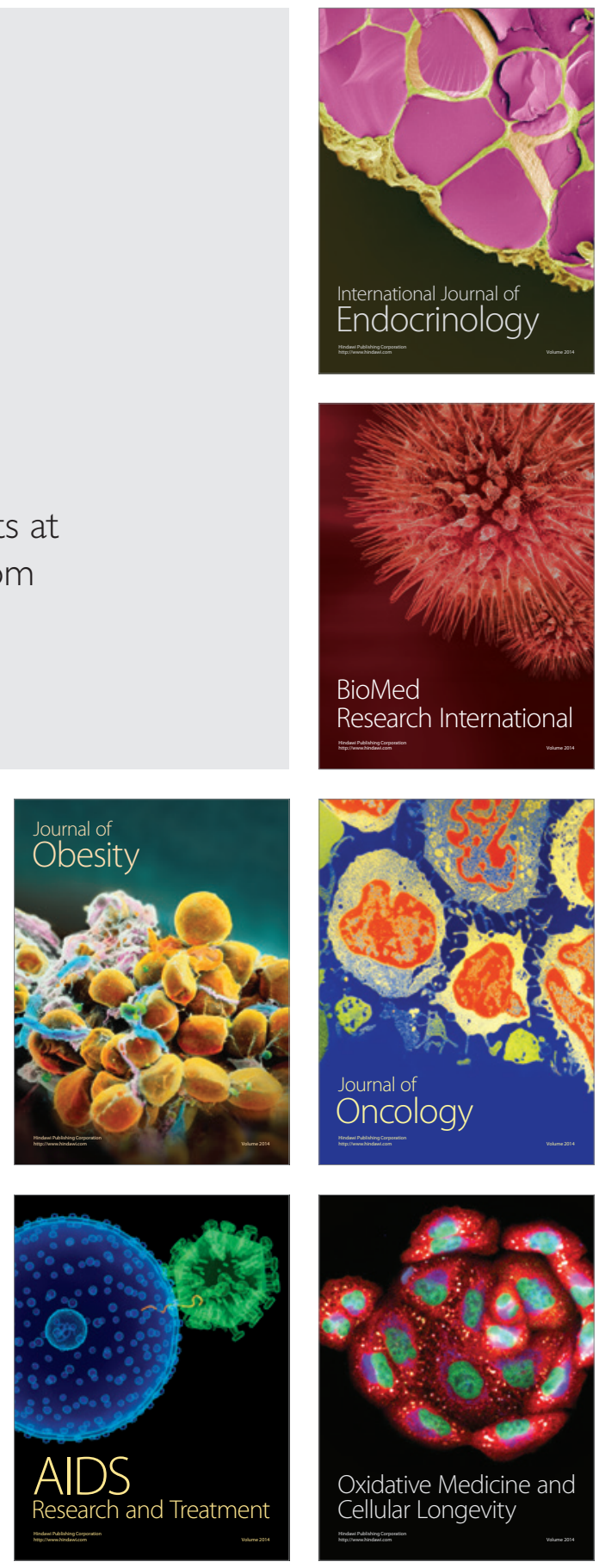